CARNETS DE Carnets de géographes

GÉOGRAPHES.

$1 \mid 2010$

Varia

\title{
Armelle Chopin et Nouakchott : du comparatisme a la monographie
}

Roman Stadnicki

\section{Q OpenEdition}

Journals

Édition électronique

URL : http://journals.openedition.org/cdg/1982

DOI : $10.4000 /$ cdg. 1982

ISSN : 2107-7266

Éditeur

UMR 245 - CESSMA

Référence électronique

Roman Stadnicki, «Armelle Chopin et Nouakchott : du comparatisme a la monographie », Carnets de géographes [En ligne], 1 | 2010, mis en ligne le 01 octobre 2010, consulté le 24 septembre 2020. URL : http://journals.openedition.org/cdg/1982 ; DOI : https://doi.org/10.4000/cdg.1982

Ce document a été généré automatiquement le 24 septembre 2020.

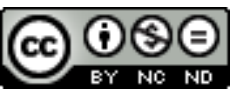

La revue Carnets de géographes est mise à disposition selon les termes de la Licence Creative Commons Attribution - Pas d'Utilisation Commerciale - Pas de Modification 4.0 International. 


\title{
Armelle Chopin et Nouakchott : du comparatisme a la monographie
}

\author{
Roman Stadnicki
}

1 Armelle Choplin livre une publication tirée de sa thèse de doctorat soutenue en 2006 et intitulée: Fabriquer des villes-capitales entre monde arabe et Afrique noire: Nouakchott (Mauritanie) et Khartoum (Soudan). Dans cette publication (Nouakchott. Au carrefour de la Mauritanie et $d u$ monde, Paris, Karthala/PRODIG, 2009), l'auteure fait le choix d'abandonner la perspective comparative qui a guidé son doctorat. Ce glissement de la comparaison à la monographie est l'objet de ce carnet de terrain qui expose dans un premier temps le contenu de l'ouvrage Nouakchott. Au carrefour de la Mauritanie et du monde avant de laisser place à un court dialogue entre la revue et l'auteur.

2 Le parti pris d'Armelle Choplin est à contre courant dans la mesure où elle fait le choix, minoritaire dans les études urbaines contemporaines, de dresser le portrait géographique d'une capitale, Nouakchott en l'occurrence, qu'elle cherche à appréhender dans sa globalité, du moins en ce qui concerne sa fabrication, son organisation et son fonctionnement. «Comment se construit désormais la ville-capitale d'un pays pauvre dans un contexte néolibéral mondialisé ?» (p. 30); tel est le projet de l'ouvrage. Il en résulte une monographie moderne et multiscalaire, particulièrement bienvenue dans un paysage éditorial assez pauvre sur la capitale mauritanienne.

Grâce à un jeu d'échelles judicieux, on passe facilement des logiques politiques locales de production d'une "capitale moderne" aux réalités de son internationalisation. L'auteur renoue même avec la notion de "carrefour ", présente dès le sous-titre de l'ouvrage, que d'aucuns ne considèrent plus que comme un concept-valise de la géographie régionale « classique ». On pourra d'ailleurs discuter la pertinence de cette notion pour décrire les relations de Nouakchott au « monde »: comme l'indiquent bien la photographie de couverture et sa légende, Doubaï et Bagdad sont d'abord, pour la ville de Nouakchott, des quartiers de sa grande banlieue. L'inscription tardive et balbutiante de la Mauritanie dans la mondialisation nous inviterait plutôt à relativiser l'intensité et l'efficacité des connexions internationales de sa capitale. Le dernier coup d'État de 2008, qui ramène les militaires au pouvoir, tout comme la baisse de la 
production pétrolière et l'irruption de la violence islamiste au cours de ces dernières années, auraient en effet tendance à renforcer l'isolement du pays, qui compte parmi les plus pauvres de la planète. L'auteur elle-même, dans son introduction, rappelle que la Mauritanie vit actuellement une période de transition sans précédent, affectant l'ensemble des structures politiques, économiques, sociales, identitaires, etc.: «Un seuil s'apprête à être franchi, une nouvelle ère s'amorce mais nul ne connaît pour l'instant la direction à prendre, ni le contenu, pas plus que l'issue » (p. 25). C'est à cette situation transitoire et à ses effets territoriaux sur la capitale mauritanienne que l'auteur consacre son analyse. Le mérite n'en est pas mince eu égard aux difficultés qu'il y a à étudier le changement dans cette ville $\mathrm{du}$ « Sud » devenue capitale politique de première importance en un demi-siècle seulement, située au contact entre Sahara et Sahel, entre l'océan Atlantique et la Trarza - région intérieure en grande partie désertique - entre ethnies Maures, Peuls et Wolofs entre autres. Situation d'interface plus que de carrefour en somme!

Dans un premier temps, l'ouvrage examine les logiques politiques de création d'une capitale ex nihilo et l'organisation de l'espace social qui en résulte. Les entrées analytiques choisies sont usuelles mais non moins efficaces: rapports entre espace projeté, espace construit et espace habité/vécu et tensions entre centre(s) et marge(s). Ce souci qu'a l'auteur d'opérer un va-et-vient permanent entre la marque du pouvoir sur le territoire nouakchottois et la réception sociale du projet urbain et des politiques urbaines, dans une vision dialectique, est présent tout au long de l'ouvrage. On apprend ainsi, dans les détails, comment et pourquoi a émergé ce pôle urbain : sa construction tardive d'abord, en 1957, dans un espace désertique très peu convoité par les Français, et son rôle d'unificateur ensuite, de la nation mauritanienne et du peuple mauritanien. Une gageure, nous dit en substance Armelle Choplin qui pointe l'incompatibilité des intérêts politiques de centralisation du pouvoir et des modes de vie des populations « dont les paradigmes demeurent la mobilité et la plasticité des découpages» (p. 60). Les défaillances de la planification territoriale, malgré la succession de schémas directeurs depuis les années 1960, et les multiples formes de fragmentation sociospatiale, auxquelles l'auteur consacre des analyses minutieuses, ne sont-elles pas des conséquences très palpables de cette incompatibilité ? Mais l'auteur ne manque pas non plus de souligner que les mécanismes ségrégatifs font partie intégrante du projet politique de "Nouakchott-capitale », cette dernière étant avant tout un vecteur de la politique d'arabisation des peuples de Mauritanie insufflée par la classe dirigeante appartenant à l'ethnie maure. L'Histoire est à ce titre constamment revisitée par le pouvoir en place, la rupture avec le monde subsaharien encouragée, les programmes scolaires modifiés et l'arabité survalorisée, dans les médias comme dans l'espace public urbain (noms des rues par exemple). C'est donc à travers le prisme des rapports de domination que l'auteur examine les morphologies socio-spatiales en présence dans le grand Nouakchott, mettant en opposition l'espace et les réseaux économico- religieux des élites maures en quête de contrôle territorial et les territoires quotidiens d'une majorité de laissés pour compte, noirs principalement, installés dans les quartiers populaires très étirés de la périphérie orientale. La vision dualiste et l'opposition radicale entre centre et périphérie est toutefois rapidement abandonnée au profit d'une analyse très convaincante du renversement progressif des polarités et des dynamiques sociales d'une société civile en émergence, preuve que rien est figé dans l'espace urbain et que la «ville-bidonville» tend à prendre le pas sur « Nouakchott-capitale » (p. 139). Le cas des Harâtîn, groupe social négro- mauritanien 
longtemps stigmatisé, aujourd'hui visiblement à l'origine d'une nouvelle forme de culture urbaine à Nouakchott, mériterait à ce titre d'être encore plus développé.

Dans un deuxième temps, l'ouvrage se consacre à l'étude de l'inscription de Nouakchott dans la mondialisation, en observant, d'une part, les effets sur le territoire et le foncier locaux de la diffusion des principes de gouvernance et des politiques néolibérales et, d'autre part, les prémisses du processus de métropolisation de la capitale mauritanienne. On appréciera la précision avec laquelle est décrypté le système d'acteurs en place, qui permet d'évaluer le rôle des acteurs exogènes, Banque mondiale, PNUD et bailleurs bilatéraux entre autres, lesquels semblent avoir pris en main le développement de la capitale. Fortement tributaire de l'aide extérieure, le gouvernement mauritanien n'est pas porteur, apprend-on, de réel projet de planification stratégique. Il se contente d'énoncer quelques règles du jeu, par le truchement d'un certain nombre d'organes déconcentrés dont la multiplication participe d'une confusion générale entre État central et pouvoirs locaux. Reste la figure omniprésente du chef de l'État, devant laquelle même la Banque mondiale semble s'effacer... Le Président actuel, exprime pour la ville un intérêt certain, en assurant notamment la maîtrise d'ouvrage du vaste Plan de Développement Urbain, qui s'échelonne sur toutes les années 2000, ainsi que la mise en œuvre d'outils de gestion foncière, qui lui permettent d'entretenir ses réseaux clientélistes. Le dernier chapitre, "Nouvelles connexions, flux globaux et tentatives d'internationalisation", pourra quant à lui déconcerter par son aspect très composite. L'auteur y range l'ensemble des indices de «l'extraversion»- terme judicieusement choisi- de la capitale mauritanienne, autrement dit ce qui en fait une ville appartenant à l'urbain mondialisé. Sont ainsi évoqués les nouveaux échanges financiers et l'influence grandissante du modèle dubaïote, les migrations transsahariennes dont Nouakchott serait une " plaque tournante ", le croisement des références bédouines et occidentales dans la citadinité locale, la menace terroriste croissante, etc. Si chacune de ces rubriques est plutôt bien renseignée, l'unique prisme de la globalisation - dont, encore une fois, les effets sur la capitale mauritanienne sont à relativiser, ce que fait d'ailleurs l'auteur elle-même dans sa conclusion - peine à créer du lien et de la cohésion.

6 Ce cinquième chapitre reflète l'ambition du projet éditorial tout en exacerbant sa principale faiblesse : une légère impression de dispersion, qui tient à la volonté de l'auteur d'aborder la situation nouakchottoise dans ses dimensions plurielles, politique, économique, sociale, spatiale, urbanistique, culturelle, etc. Nouakchott, Au carrefour de la Mauritanie et du monde s'impose désormais comme une véritable mine dont les futurs chercheurs attirés par la Mauritanie ne pourront plus se passer. Armelle Choplin signe donc là un ouvrage clé pour comprendre les dynamiques urbaines contemporaines qui agitent la capitale mauritanienne. L'aller et retour entre logiques institutionnelles et dynamiques sociales, rendu possible grâce à une très bonne connaissance du terrain, fonctionne bien. Au-delà du cas nouakchottois, l'ouvrage éclairera tous ceux qui s'intéressent au renouvellement des modalités de l'action publique et de la fabrication urbaine dans une métropole "incomplète" mais pas totalement déconnectée des grands courants d'échanges internationaux.

\section{Entretien de Roman Stadnicki avec Armelle Choplin}


dans Votre ouvrage, Le choix de la monographie ReMet-IL EN QUestion La PERTINENCE de L'APPROCHE COMPARATIVE dÉFENDUE dANS VOTRE THÈse ?

Armelle Choplin: l'approche comparative fut d'une grande richesse. J'avais déjà conduit des recherches sur Nouakchott dans le cadre de ma maîtrise et de mon DEA. Aussi, dès le début du doctorat, je songeais à élargir mes horizons. Choisir un second terrain, c'était également un moyen de me préserver : la Mauritanie commençait à prendre trop de place dans ma vie. Aussi, lorsque je décidais de travailler sur le Soudan et de me rendre à Khartoum à la fin de ma première année de thèse, ce fut comme une bouffée d'air, un nouveau départ. En retournant à Nouakchott quelques mois plus tard, j'ai redécouvert la ville. Le décentrage soudanais m'a réellement éclairée sur la capitale mauritanienne. En réalité, c'est lors de mon second séjour au Soudan que je crois avoir compris Nouakchott. Les trajectoires de ces deux villes se faisaient écho. Mes choix également. La comparaison me permettait de prendre du recul, de déceler des dynamiques générales, observables partout, et parallèlement, de mettre en exergue l'originalité de chaque espace urbain. Si je n'avais eu l'occasion d'étudier Khartoum, j'aurais certainement fait une monographie assez simpliste de Nouakchott.

Mais, ce travail comparatif a créé une surcharge de travail énorme (nouvelle bibliographie, nouveau terrain, nouveaux contacts...). Bref, un lourd investissement dont je doute parfois de la "rentabilité». On ne peut prétendre connaître les deux cas étudiés de la même façon, l'un demeurant plus familier que l'autre. Finalement, je me demande si les "approches comparées" qui sont à la mode depuis quelques années en géographie ne ressemblent pas davantage à de bons cas d'étude éclairés de temps à autre par un exemple approfondi.

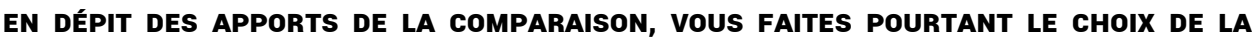 MONOgRAPHIE... POURQUOI ?}

Armelle Choplin: au lendemain de ma thèse, différents programmes de recherche et une actualité sensible m'ont ramené à Nouakchott. J'ai pris plaisir à réétudier la ville sous d'autres angles et à approfondir certains points que, du fait de la comparaison, j'avais laissés en suspens. Au final, mes récents séjours m'ont permis d'acquérir une bonne connaissance de la ville, d'où le retour à la monographie et l'écriture de l'ouvrage. Il faut préciser ici que j'ai eu la chance d'étudier l'une de ces dernières petites capitales du monde. Nouakchott est encore à " taille humaine ", ce qui signifie pour le chercheur la possibilité d'observer dans son ensemble des mutations qui ailleurs sont plus diluées. Alors, oui, d'une certaine façon, je crois au retour de la monographie dite "classique" en géographie. Dans ces lieux un peu oubliés, la monographie permettrait de saisir, peut-être moins "mal» qu'ailleurs, une partie de la complexité du monde contemporain.

\section{INDEX}

Thèmes : Carnets de lectures 
AUTEUR

ROMAN STADNICKI

Post-doc CITERES-EMAM 